\title{
Editorial
}

Chemotherapy

Chemotherapy $2021 ; 66: 1-2$

Received: 04 19, 2021

DOI: 10.1159/000516594

Accepted: 04 19, 2021

Published online: May 25, 2021

\section{Quo Vadis COVID? Lessons from a Focus Topic Issue of Chemotherapy}

\author{
Giorgio Minotti \\ Department of Medicine, University Campus Bio-Medico of Rome, Rome, Italy
}

The editors present a special focus on COVID-19-related articles in this issue of the journal Chemotherapy. Five articles have been selected for this issue. Two of them deal with SARS-CoV-2 variants and sublineages that have been identified in Italy or worldwide. Three other articles deal with diagnostics issues, development of animal models for devising strategies against infection, original insights into the role of microbiomes and the gut-lung axis in the pathogenesis of SARS-CoV-2-related pneumonia. With this issue, the journal Chemotherapy contributes to a much needed mechanism-based debate on COVID-19.

COVID-19 has changed everything, from our lifestyle to our perception of modern medicine. Some of us witnessed the pain and difficult times our beloved ones had to go through in their fight against the disease. All of us suddenly realized how unprepared our healthcare systems were to manage a pandemic. After all, we must confess that our idea of pandemic was in fact quite naive and out of context. In our language, the word "pandemic" was an elegant epidemiologic definition to indicate a highly prevalent disease, for example, diabetes, a worldwide circulating virus being definitely out of our medical landscape and personal imagination.

COVID-19 has changed the publication system as well. Some 58,000 COVID-19 articles can now be found in PubMed, which intuitively describe the immense and not always tightly controlled interest the journals developed in this subject matter. In very many respects, a new

karger@karger.com

(c) 2021 S. Karger AG, Basel

www.karger.com/che

Karger" pandemic came to stage, we may call it publication bulimia. This is especially true for articles on how COVID-19 should be prevented or treated as the lack of knowledge we had about the virus prompted very many scientists to elaborate and submit the most extravagant ideas. And the journals were greedy for publishing COVID-19-related papers, no matter how robust and reproducible the ideas behind the submissions were. In times when the credibility of a journal binds to magic numbers, the impact factor being the master of them all, publishing on COVID-19 was the obvious way to increasing citations and climbing the rank of journal metrics. We at Chemotherapy were not so generous, we did receive COVID-19-related papers, but the editors and reviewers recommended only few of them for publication. But the few manuscripts we published turned out to be highly cited or accessed by visitors to our Journal Web site. Could have we published more? Was our editorial policy too stringent in times of publication pandemic? We do not have a foundation to say yes or no at this point in time. We will know in few months when our yearly school report, impact factor, will be published. Meanwhile, everybody wonders how long the pandemic will last and how long the journals will have to manage waves of solid, sound, or bizarre articles on COVID-19.

Here, we would like to introduce our readers to a special focus on COVID-19 in this issue of Chemotherapy. It is not a twist in our strategy; it is the result of our thought- 
fully inviting some established leaders in the field to submit what they really deem important and worth sharing with our readers. There is a logic in the string of articles we publish. The contribution by Benvenuto et al. [1] and Cella et al. [2] deals with SARS-CoV-2 variants and sublineages that have been identified in Italy or circulate worldwide. Needless to say, both articles are important for updating diagnostic tools and surmising new avenues to disease prevention by vaccination, better care, and timely treatment. The article by Ciotti et al. [3] then represents a timely contribution on the development of reliable diagnostic assays and their impact on decision-making processes, including interpretation of serological assays to track the efficacy of vaccination or therapy with monoclonal antibodies. But our fight against COVID-19 begins in the laboratory, and so the special focus offers two more contributions on translationally oriented research. Parolin et al. [4] describe the role of animals as reservoirs and natural hosts of SARS-CoV-2, and the importance that animal models for COVID-19 may have to design countermeasures against SARS-CoV-2 infection.
And finally, Spagnolello et al. [5] build on what we currently know about the gut-lung axis to foresee the beneficial role that correcting intestinal dysbiosis may have on mitigating immune-related determinants of pneumonia.

We hope this special issue of Chemotherapy will serve a proper blend of facts and hypotheses about COVID-19. The floor is to our readers. We the editors strive to improve the quality of articles published in the Journal, but needless to say, only comments and feedbacks from you the readers will give us an idea of how correct our choices are.

\section{Giorgio Minotti, Editor-in-Chief}

\section{Conflict of Interest Statement}

This editorial does not have conflict of interest to declare.

\section{Funding Sources}

There was no finding support to the writing of this editorial.

\section{References}

1 Benvenuto D, Benedetti F, Demir AB, Ciccozzi M, Zella D. Analysis of three mutations in Italian strains of SARS-CoV-2: implications for pathogenesis. Chemotherapy. 2021.

2 Cella E, Benedetti F, Fabris S, Borsetti A, Pezzuto A, et al. SARS-CoV-2 lineages and sublineages circulating worldwide: a dynamic overview. Chemotherapy. 2021.
3 Ciotti M, Benedetti F, Zella D, Angeletti S, Ciccozzi M, Bernardini S. SARS-Cov-2 infection and the COVID-19 pandemic emergency: the importance of diagnostic methods. Chemotherapy. 2021.

4 Parolin C, Virtuoso S, Giovanetti M, Angeletti S, Ciccozzi M, Borsetti A. Animal hosts and experimental models of SARS-CoV-2 infection. Chemotherapy. 2021.
5 Spagnolello O, Pinacchio C, Santinelli L, Vassalini P, Innocenti GP, et al. Targeting microbiome: an alternative strategy for fighting SARS-Cov-2 infection. Chemotherapy. 2021. 